\title{
A survey of T Tauri stars with AKARI towards the Taurus-Auriga region ${ }^{\star}$
}

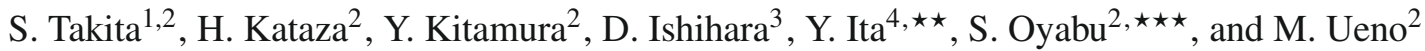 \\ 1 Department of Space and Astronautical Science, The Graduate University for Advanced Studies (Sokendai), 3-1-1 Yoshinodai, \\ Chuo, Sagamihara, Kanagawa 252-5210, Japan \\ e-mail: takita@ir.isas.jaxa.jp \\ 2 Institute of Space and Astronautical Science, Japan Aerospace Exploration Agency, 3-1-1 Yoshinodai, Chuo, Sagamihara, \\ Kanagawa 252-5210, Japan \\ 3 Division of Particle and Astrophysical Sciences, Nagoya University, Furo-cho, Chikusa-ku, Nagoya 464-8602, Japan \\ ${ }^{4}$ National Astronomical Observatory of Japan, 2-21-1 Osawa, Mitaka, Tokyo 181-8588, Japan
}

Received 15 October 2009 / Accepted 1 June 2010

\section{ABSTRACT}

Context. The first Japanese infrared astronomical satellite, AKARI, has completed an All-Sky Survey at mid- to far-infrared wavelengths with higher spatial resolutions and sensitivities than the previous survey with Infrared Astronomical Satellite (IRAS).

Aims. We search for new T Tauri star (TTS) candidates with the mid-infrared (MIR) part of the AKARI All-Sky Survey at 9 and $18 \mu$ m wavelengths.

Methods. We used the point source catalogue (PSC) obtained by the Infrared Camera (IRC) on board AKARI. We combined the 2MASS PSC and the 3rd version of the USNO CCD Astrograph Catalogue (UCAC) with the AKARI IRC-PSC, and surveyed 517 known TTSs over a 1800-square-degree part of the Taurus-Auriga region to develop criteria to extract TTSs. We considered asymptotic giant branch (AGB) stars, post-AGB stars, planetary nebulae (PNe), and galaxies, which have similar MIR colours, to separate TTSs from these sources. We finally searched for new TTS candidates from AKARI IRC-PSC in the same Taurus-Auriga region.

Results. Of the 517 known TTSs, we detected 133 sources with AKARI: 46 sources were not detected by IRAS. Based on the colour-colour and colour-magnitude diagrams made from the AKARI, 2MASS, and UCAC surveys, we propose the criteria to extract TTS candidates from the AKARI All-Sky data, and 68/133 AKARI detected TTSs have passed these criteria. On the basis of our criteria, we selected 176/14725 AKARI sources as TTS candidates that are located around the Taurus-Auriga region. Comparing these sources with SIMBAD, we found that 148 are previously identified sources including 115 young stellar objects (YSOs), and 28 unidentified sources.

Conclusions. Based on SIMBAD identifications, we infer the TTS-identification probability using our criteria to be $\sim 75 \%$. We find 28 TTS candidates, of which we expect $\sim 21$ to be confirmed once follow-up observations can be obtained. Although the probability of $\sim 75 \%$ is not so high, it is affected by the completeness of the SIMBAD database, and we can search for TTSs over the whole sky, and all star-forming regions.

Key words. stars: formation - stars: late-type - stars: pre-main sequence - infrared: stars

\section{Introduction}

T Tauri stars (TTSs) are low-mass pre-main sequence (PMS) stars with ages of $\sim 10^{6}-10^{7}$ yrs (Adams et al. 1988). TTSs were traditionally identified with the strong $\mathrm{H} \alpha$ emission line originating in their natal molecular clouds, i.e., classical TTSs (CTTSs) with ages of $\sim 10^{6}$ yr. On the other hand, X-ray observations, such as the Einstein Observatory and ROSAT surveys, have discovered another class of PMS stars

* Tables 1 and 2 are only available in electronic form at the CDS via anonymous ftp to cdsarc.u-strasbg. fr (130.79.128.5) or via http://cdsarc.u-strasbg.fr/viz-bin/qcat?J/A+A/519/A83

$\star \star$ Present address: Astronomical Institute, Graduate School of Science, Tohoku University, 6-3 Aramaki, Aoba-ku, Sendai 980-8578, Japan.

$\star \star \star$ Present address: Division of Particle and Astrophysical Sciences, Nagoya University, Furo-cho, Chikusa-ku, Nagoya 464-8602, Japan. (e.g., Neuhäuser et al. 1995a). Since these sources have "weak" $\mathrm{H} \alpha$ emission lines with equivalent widths of $\leq 10 \AA$, they are called weak-line TTSs (WTTSs) and have ages of $\sim 10^{7} \mathrm{yr}$. The youth of WTTSs was confirmed by strong Li I absorption lines, since lithium is easily destroyed in the stellar atmosphere at high temperature. In contrast to the CTTS case, many WTTSs are found outside the molecular clouds. This situation is interpreted as follows: the natal molecular clouds were dispersed at $\sim 10^{7} \mathrm{yr}$, or the stars left the natal clouds because of their motions of a few mas/yr.

The circumstellar disks around TTSs are assumed to be the birthplaces of planets. The pioneering studies of the disks are the IRAS and millimetre continuum surveys towards the Taurus-Auriga region (Strom et al. 1989; Beckwith et al. 1990). Strom et al. (1989) found that about a half of all CTTSs have excess emission at infrared (IR) wavelengths stronger than that expected from their photospheres. The excess emission can 
be attributed to thermal emission from the circumstellar disks heated by the central stars and/or mass accretion. On the other hand, most WTTSs lack this excess emission. Even IR observations with the Spitzer Space Telescope have confirmed the weakness of the excess emission towards WTTSs. Silverstone et al. (2006) found that 5 of 74 young stars (3-30 Myr) have strong IR excess, but have spectral energy distributions consistent with CTTSs. Padgett et al. (2006) observed 83 WTTSs outside their natal clouds, and found that only 5 of the WTTSs have excess emission. The largest Spitzer survey of WTTSs completed so far was that of Cieza et al. (2007), which observed more than 230 WTTSs located in the Ophiuchus, Lupus, and Perseus molecular clouds. Their data indicate that $\sim 20 \%$ of the WTTSs have IR excess emission, but no IR excess for the stars older than 10 Myr. It is therefore most likely that the disk dissipation time-scale is about 10 Myr. However, IRAS was of insufficient sensitivity to detect the excess emission of WTTSs, and Spitzer cannot cover "all" WTTSs. Therefore, we need unbiased and high-sensitivity surveys to study the WTTS disks with good statistics.

\section{AKARI all-sky data}

\subsection{AKARI IRC All-Sky Survey}

AKARI is the first Japanese infrared astronomical satellite dedicated to infrared astronomy (Murakami et al. 2007). One of the major observation programs of AKARI is an All-Sky Survey at the mid- to far-infrared wavelengths with 6 photometric bands. AKARI has a higher sensitivity, a higher spatial resolution, and a wider wavelength coverage than those of the previous IRAS survey. The mid-infrared (MIR) survey has been carried out with the $S 9 W(9 \mu \mathrm{m})$ and $L 18 W(18 \mu \mathrm{m})$ bands using the Infrared Camera (IRC; Onaka et al. 2007). The $5 \sigma$ detection limit for a point source is estimated to be 50 and $120 \mathrm{mJy}$ in the $S 9 \mathrm{~W}$ and $L 18 W$ bands, respectively. The spatial resolution is about $5^{\prime \prime}$. More than $96 \%$ of the entire sky has been observed with the two bands. The first version of the AKARI IRC point source catalogue (hereafter IRC-PSC) was publicly released in March 2010. The analysis of this paper is based on the 1st version of the IRCPSC. The details of the AKARI IRC All-Sky Survey and its data reduction processes are described in Ishihara et al. (2010).

\subsection{Comparison with the NIR 2 MASS and optical UCAC catalogues}

We compared the IRC-PSC with the PSC of the near-infrared (NIR) survey, 2MASS (Skrutskie et al. 2006), using a simple positional correlation method. We used a positional tolerance of 5", which is the spatial resolution of the AKARI IRC All-Sky Survey. More than $99 \%$ of the AKARI sources can be identified with those in the 2MASS PSC to within the accuracy. We also compared the IRC-PSC with the 3rd version of the USNO CCD Astrograph Catalogue (UCAC) compiled for an optical survey (Zacharias et al. 2010) in the same way. About $70 \%$ of the AKARI sources have optical counterparts to within the $5^{\prime \prime}$ accuracy. This relatively low cross-identification rate is caused by the limited magnitude range of the UCAC survey in which the most nearby $(<100 \mathrm{pc})$ stars are saturated, and distant $(>1 \mathrm{kpc})$ or heavily reddened stars have no UCAC entry because of the catalogue's limited sensitivity. Since the UCAC catalogue contains "stars" with the magnitude range of $R=7.5-16.3$ in a $579-642 \mathrm{~nm}$ band, it is useful to search stars at distances of about $100 \mathrm{pc}$, which is the typical distance to nearby molecular clouds (for example, if we place the Sun at $140 \mathrm{pc}$, the magnitude becomes $\sim 11$ ).

\section{AKARI IRC observations of the previously known $T$ Tauri stars in the Taurus-Auriga region}

\subsection{Previously known members in the Taurus-Auriga region}

For this study, we selected a 1800 -square-degree $\left(2^{\mathrm{h}} 40^{\mathrm{m}}<\right.$ RA $<5^{\mathrm{h}} 40^{\mathrm{m}}$ and $0^{\circ}<$ Dec $<40^{\circ}$ ) part of the Taurus-Auriga region, which is a well-studied region of low-mass star formation at a close distance of $\sim 140 \mathrm{pc}$ with hundreds of pre-mainsequence (PMS) stars. We first gathered known Taurus PMS members from Rebull et al. (2010), Kenyon et al. (2008), Güdel et al. (2007), Beckwith et al. (1990), and Strom et al. (1989), associated with the Taurus molecular cloud. Secondly, we selected the following TTSs from the PMS objects: (1) Class II/III objects including the "new", "probable", and "possible" members in Rebull et al. (2010); (2) objects listed in Kenyon et al. (2008) that were confirmed as Class II/III objects on the basis of the SIMBAD database and their references; (3) objects labelled as CTTSs or WTTSs in Güdel et al. (2007). We note that Beckwith et al. (1990) and Strom et al. (1989) listed only TTSs. Furthermore, we added TTSs discovered by the Roentgen satellite (ROSAT) and distributed from the cloud (Li \& Hu 1998; Magazzù et al. 1997; Wichmann et al. 1996). In our TTS list, we considered close $\left(<5^{\prime \prime}\right.$; the spatial resolution of the AKARI IRC All-Sky Survey) binaries as a single source. We finally obtained a catalogue of 517 TTSs in the selected region. The distribution of our input TTSs is shown in Fig. 1.

\subsection{Statistical properties of the previously known T Tauri stars detected with AKARI}

We reliably detected 133 TTSs with AKARI within the positional accuracy of $5^{\prime \prime}$, i.e., the resolution of the AKARI IRC AllSky Survey: 127 and 95 sources were detected in the $S 9 \mathrm{~W}$ and $L 18 \mathrm{~W}$ bands, respectively. Six sources were detected only in the L18W band: 3 sources (IRAS 04200+2759, IRAS 04295+2251, and UX Tau) were observed/detected only once in the $S 9 W$ band, so the IRC-PSC does not contain these sources, and the other 3 sources were not detected at all. The positions of almost all the AKARI detected sources agree well with those in the previous catalogues to within the uncertainty of $3^{\prime \prime}$ (see Fig. 2). Although the positions of GI Tau in the different catalogues have larger differences than $3^{\prime \prime}$, they still agree to within the positional accuracy of AKARI IRC-PSC. The photometric data of the AKARI detected TTSs are listed in Table 1.

In the 133 TTSs, 46 faint sources have no counterparts in the IRAS PSC (FQUAL12 $=3$ or FQUAL25 $=3$ ) with a searching radius of $60^{\prime \prime}$, the IRAS resolution, as shown in Fig. 3. These "new detections" are achieved because of the higher sensitivity and spatial resolution of AKARI compared to IRAS. Figure 3 shows that the detection limit for the previously known Taurus TTSs in the $S 9 W$ band has been improved: IRAS was able to detect almost all the TTSs with brighter $S 9 W$ and $L 18 W$ band magnitudes than 6 and 4, respectively, but only about a quarter of the fainter sources were detected with IRAS. Figure 4 shows the $\left(K_{\mathrm{S}}-S 9 W\right)$ vs. $(S 9 W)$ colour-magnitude diagram of the AKARI detected sources. Since about half of the sources that were not detected with IRAS have relatively blue colours with $K_{S}-S 9 W<1$ and most of the IRAS-detected TTSs have, in contrast, relatively red colours with $K_{\mathrm{S}}-S 9 W>2$, a gap appears to exist at $K_{\mathrm{S}}-S 9 W \sim 1$. The sources with $K_{\mathrm{S}}-S 9 W<1$ 


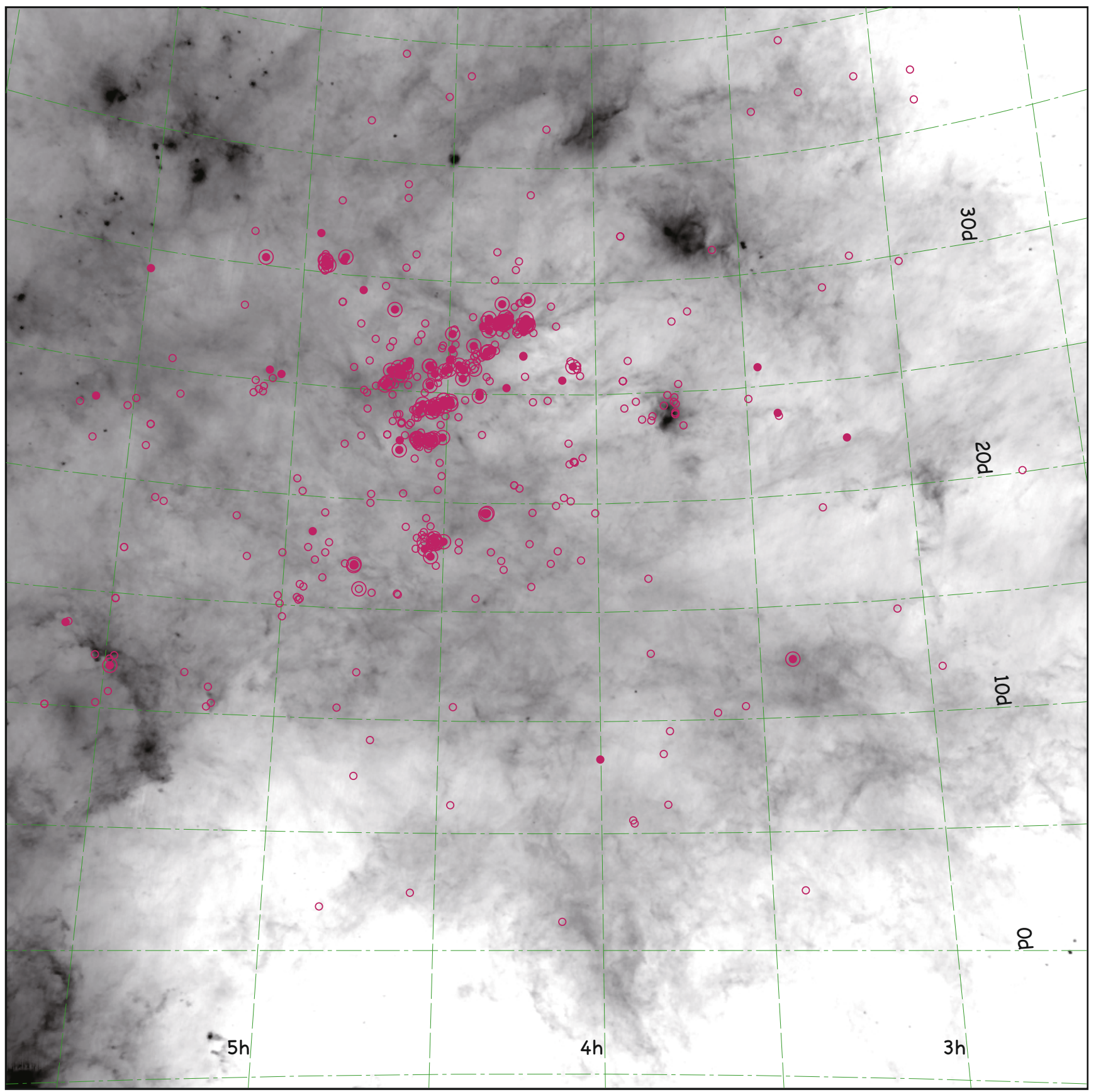

Fig. 1. Distribution of the target TTSs (small open circles). The small filled circles indicate the TTSs detected with AKARI and the large open ones represent the IRAS detection, on the IRAS $100 \mu \mathrm{m}$ map.

are most likely to be WTTSs, because they have weak $\mathrm{H} \alpha$ emission (except HT Tau, whose $\mathrm{H} \alpha$ equivalent width is not given) and are located near the periphery of the clouds or outside the clouds. AKARI has succeeded in detecting WTTSs with weak excess emission in the sensitive $S 9 W$ band.

Eighteen TTSs are not catalogued in the IRC-PSC to within a $5^{\prime \prime}$ searching radius, but are catalogued in the IRAS PSC to within $60^{\prime \prime}$ radius. Of these sources, 15 sources have AKARI counterparts within the positional accuracy of the IRAS, but the remaining 3 sources have no counterparts in the IRC-PSC. IRAS $04302+2247$ was observed once and four times at the S9W and $L 18 W$ bands, respectively, but not detected. Although this source is a well-known TTS with an edge-on disk, it is faint in the $S 9 \mathrm{~W}$ and $L 18 \mathrm{~W}$ bands, which suggests that there is an inner gap in the disk. It has indeed a bright $24 \mu \mathrm{m}$ magnitude of 3.57 but faint $8 \mu \mathrm{m}$ one of 9.71 (Rebull et al. 2010). IRAS $04216+2603$ was observed four times and detected only once at the $L 18 \mathrm{~W}$ band, and not observed in the $S 9 \mathrm{~W}$ band at all. Since the IRC-PSC should contain more than one detection of a "real" point source to reject a moving object or a fake one, the source is not catalogued in the IRC-PSC. Finally, DM Tau was detected twice in the $L 18 \mathrm{~W}$ band, but the positions in the two independent images have a larger difference than $5^{\prime \prime}$ between each other for the current positional accuracy, i.e., no entry in the IRC-PSC.

Although Spitzer was not used to survey the entire region we searched, sources inside the Taurus molecular cloud and some other sources outside the cloud have also been observed with 


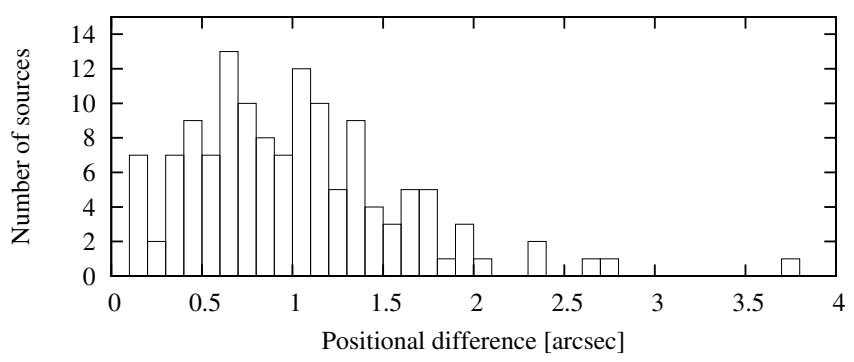

Fig. 2. Histogram of the positional differences between the TTSs detected with AKARI in the IRC-PSC and those in the previous catalogues. The size of the positional difference bin is $0.1 \mathrm{arcsec}$.
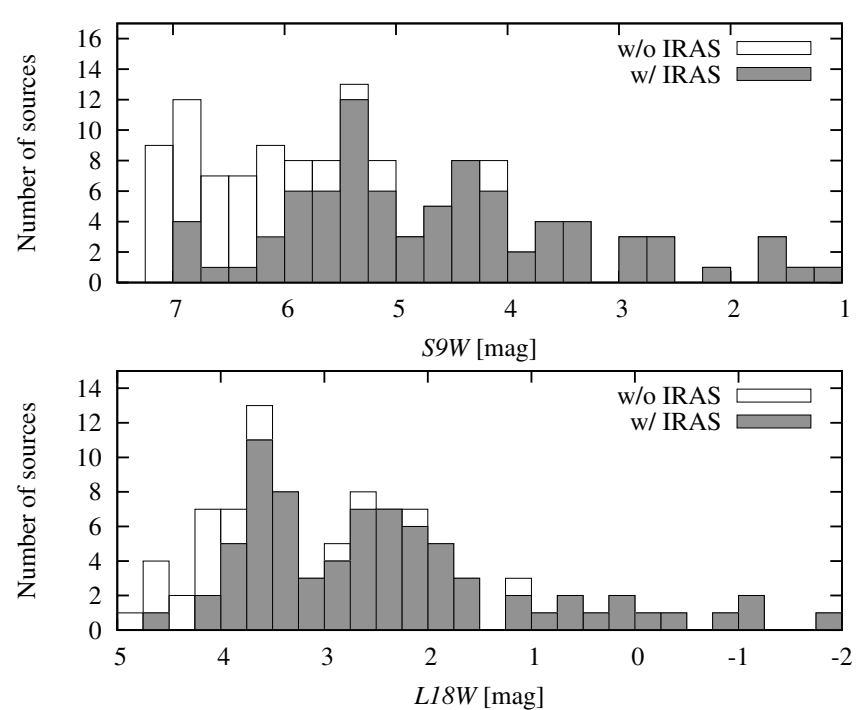

Fig. 3. Histogram of the $S 9 W$ (top) and L18W (bottom) magnitudes of the TTSs detected with AKARI. The filled and open bars indicate the sources that were detected and not detected with IRAS, respectively. The size of the magnitude bin is 0.25 mag.

Spitzer (e.g., Rebull et al. 2010). Since Spitzer has higher sensitivity than the AKARI All-Sky Survey, Spitzer has discovered more faint sources as shown in Fig. 5, which shows the histogram of the IRAC4 $(8 \mu \mathrm{m})$ magnitudes of the detected TTSs with Spitzer. The AKARI All-Sky Survey should be able to detect more than $\sim 90 \%$ of the sources of magnitude brighter than 7.5 in the IRAC4 band, which agrees well with the detection limit of the AKARI $S 9 W$ band.

\section{Selection of AKARI TTS candidates}

In this section, we discuss methods for extracting TTS candidates from the AKARI All-Sky data by comparing previously known TTSs with contaminating samples.

\subsection{Other types of sources in the whole sky}

Since the following types of sources are known to have similar colours to those of the TTSs, we should be able to determine the colour properties of the sources. We considered the four additional catalogues of (1) asymptotic giant branch (AGB) stars of 126 carbon and 563 OH/IR stars (Le Bertre et al. 2003), (2) 326 post-AGB stars (Szczerba et al. 2007), (3) 1143 planetary

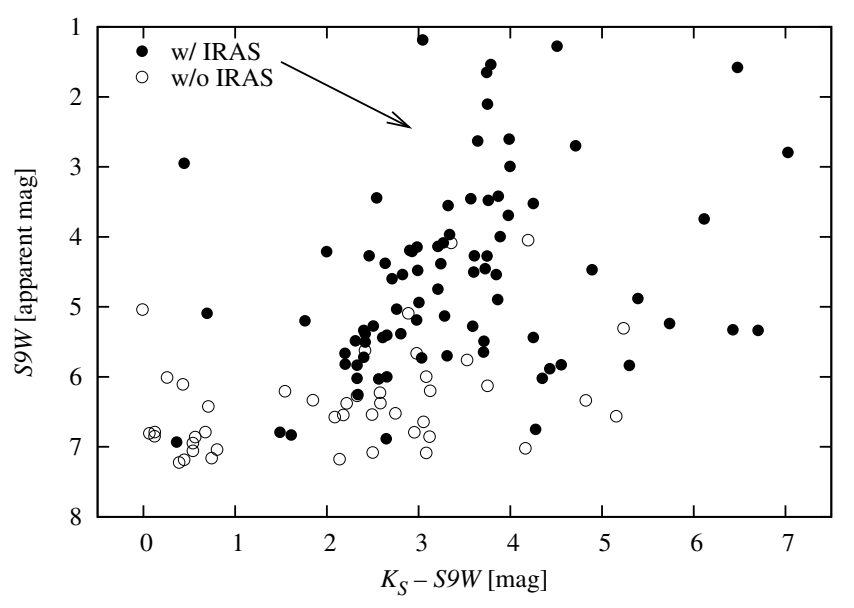

Fig. 4. $\left(K_{\mathrm{S}}-S 9 W\right)$ vs. $(S 9 W)$ colour-magnitude diagram of the AKARI-detected PMS stars. The filled and open circles indicate the sources that were detected and not detected with IRAS, respectively. The arrow shows the interstellar extinction vector of $A_{V}=20$ mag, using the Weingartner \& Draine (2001) Milky Way model of $R_{V}=3.1$.

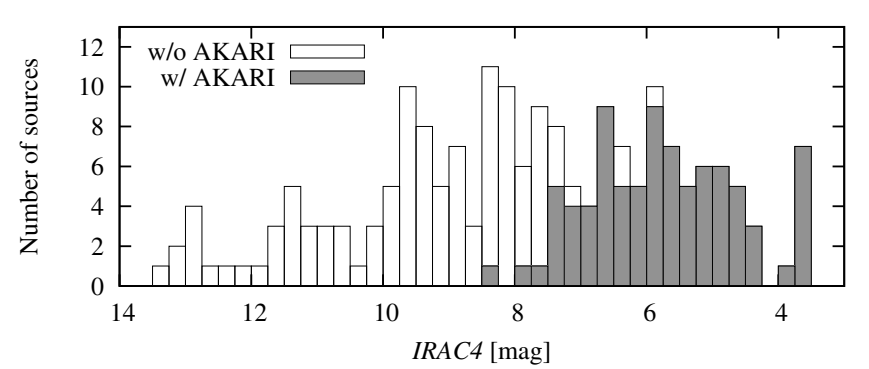

Fig. 5. Histogram of the IRAC4 magnitudes of the PMS stars listed in Table 4 by Rebull et al. (2010). The filled and open bars indicate the sources that were detected and not detected with AKARI, respectively. The size of the magnitude bin is 0.25 mag.

nebulae (PNe) (Acker et al. 1994), and (4) 2907 extragalactic objects of brighter flux density than 100 mJy in the IRAS $12 \mu \mathrm{m}$ band (the NASA Extragalactic Database). In contrast to the TTS case, we examined these sources in the whole sky because of a small number of the sources towards the Taurus-Auriga region.

\subsection{Colour-colour diagrams}

Figure 6 shows the $\left(K_{\mathrm{S}}-S 9 W\right)$ versus (vs.) $\left(J-K_{\mathrm{S}}\right)$ and $\left(K_{\mathrm{S}}-L 18 W\right)$ vs. $\left(J-K_{\mathrm{S}}\right)$ colour-colour diagrams, where the $S 9 W$ and $L 18 W$ excess emission can be clearly recognized. One object, 1RXS J032409.7+123745, does not seem to have excess emission in both the $S 9 \mathrm{~W}$ and $L 18 \mathrm{~W}$ bands among the TTSs in the Taurus-Auriga region. Although this source is listed as a WTTS with spectral type of K2 (Li \& Hu 1998), it seems to be a Li-rich giant star because of its high luminosity $(J \sim 4$ and $V \sim 6$ ), which is not expected for a $\mathrm{K} 2$ dwarf star at a distance of $\sim 140 \mathrm{pc}$. On the other hand, the other types of the sources, which are stars surrounded with dust, also have significant IR excess emission. Therefore, we need to separate these sources from TTSs in the IRC-PSC.

We first separate almost all carbon and $\mathrm{OH} / \mathrm{IR}$ stars from the TTSs in the $(S 9 W-L 18 W)$ vs. $\left(J-K_{\mathrm{S}}\right)$ colour-colour diagram 

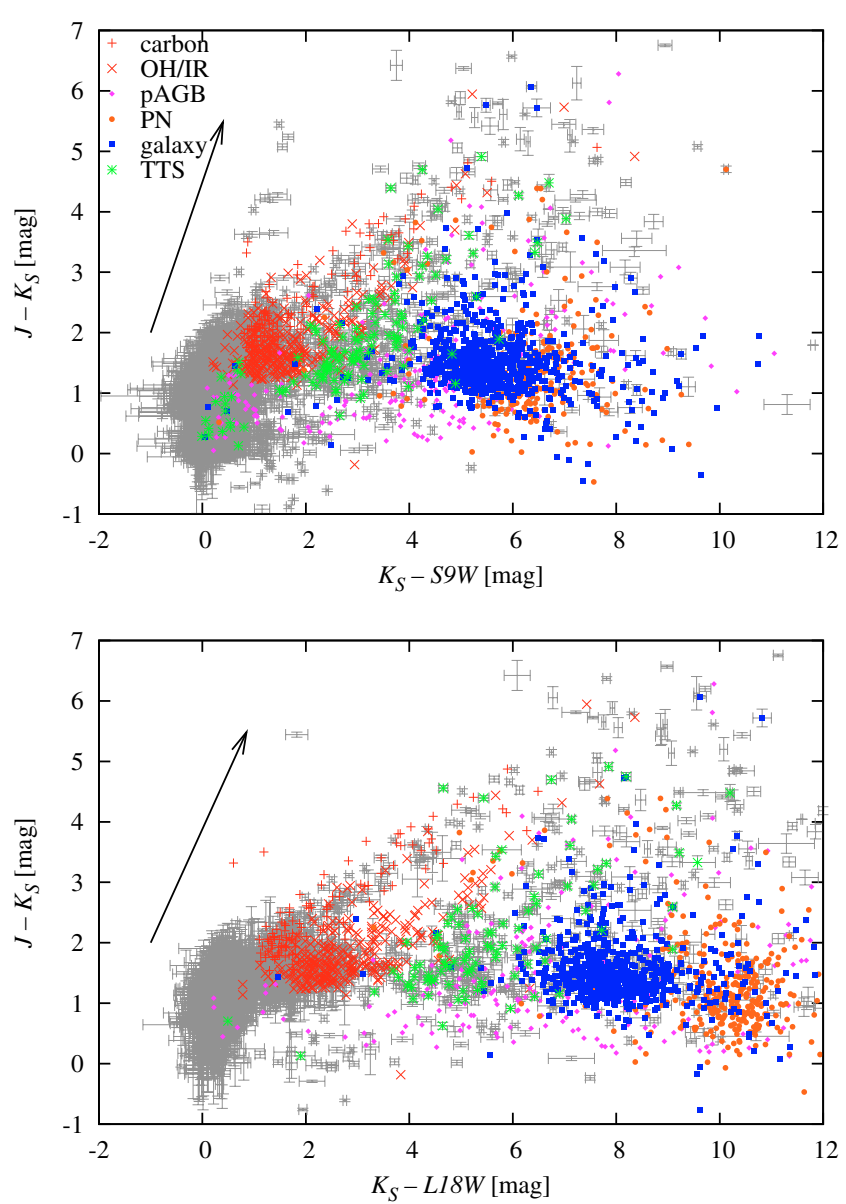

Fig. 6. Top: $\left(K_{\mathrm{S}}-S 9 W\right)$ vs. $\left(J-K_{\mathrm{S}}\right)$ colour-colour diagram. The grey dots with error bars indicate all the AKARI point sources in the selected Taurus-Auriga region. The red plus and cross symbols show carbon and $\mathrm{OH} / \mathrm{IR}$ stars in the whole sky, respectively. The magenta diamonds, the orange circles, and the blue squares are post-AGB stars, PNe, and extragalactic objects in the whole sky, respectively. The green stars mean the TTSs in the Taurus-Auriga region. The arrow shows the interstellar extinction vector of $A_{V}=20 \mathrm{mag}$, estimated from the Weingartner \& Draine (2001) Milky Way model of $R_{V}=3.1$. Bottom: $\left(K_{\mathrm{S}}-L 18 W\right)$ vs. $\left(J-K_{\mathrm{S}}\right)$ colour-colour diagram. The symbols and arrow are the same as in the top panel.

shown in Fig. 7. Since an AGB star typically has hot dust near its photosphere, the $(S 9 W-L 18 W)$ colour, which represents the dust temperature, becomes blue. On the other hand, since the majority of the dust around a TTS is cold, the MIR colour is red. To remove about 80 and $70 \%$ of carbon and OH/IR stars, we propose the first criterion to be

$$
J-K_{\mathrm{S}} \leq 7.7 \times(S 9 W-L 18 W)-8.5 \text {. }
$$

However, post-AGB stars and PNe have the red MIR colour because they have cold dust. Furthermore, galaxies also contain cold dust. Therefore, we can separate only $15 \%$ of post-AGB stars, and a few PNe and galaxies from TTSs. We note that this criterion is valid for the sources that were detected in both the $S 9 W$ and $L 18 W$ bands. We do not remove the sources that were detected in only one band.

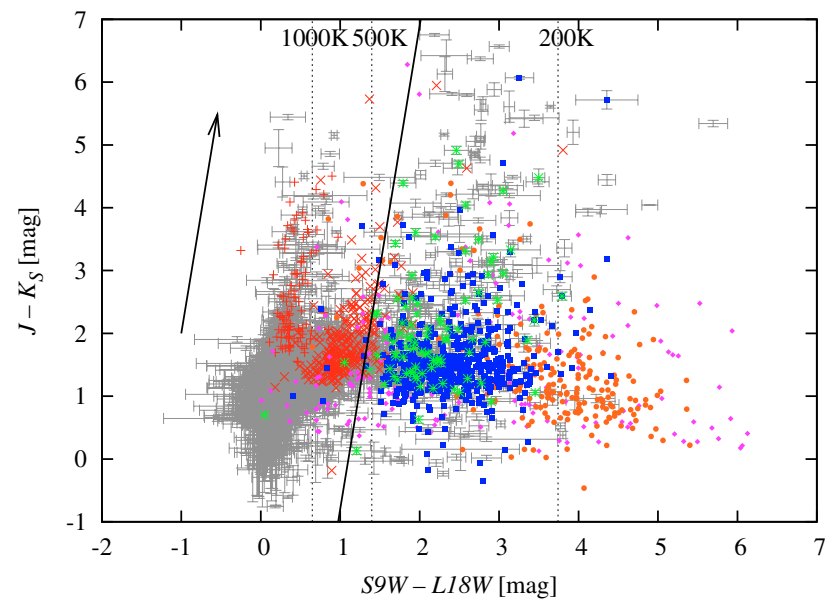

Fig. 7. $(S 9 W-L 18 W)$ vs. $\left(J-K_{S}\right)$ colour-colour diagram. The symbols and arrow are the same as in Fig. 6. The black solid line indicates our criterion (1). The black broken lines indicate $S 9 W-L 18 W$ colours of 200,500 , and $1000 \mathrm{~K}$ blackbody.

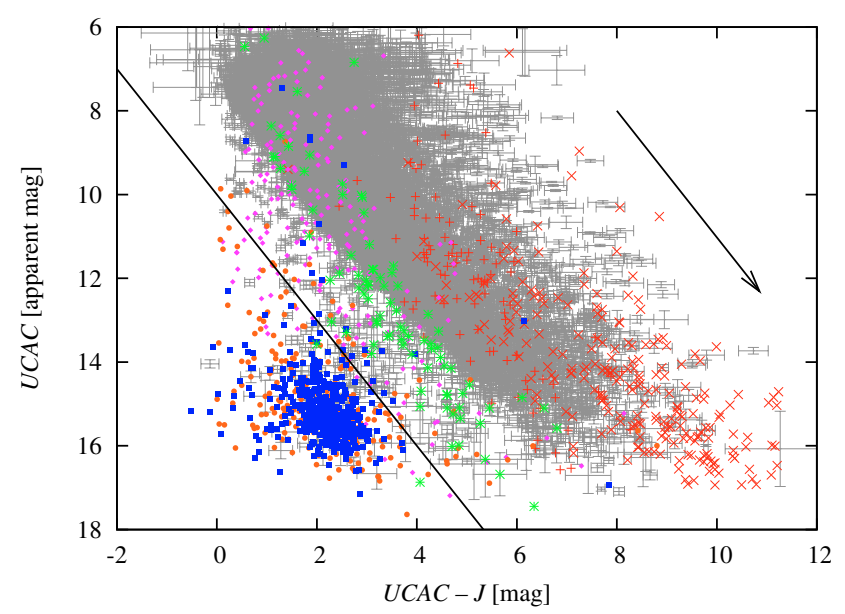

Fig. 8. (UCAC $-J$ ) vs. (UCAC) colour-magnitude diagram. The symbols are the same as in Fig. 6. The arrow shows the interstellar extinction vector of $A_{V}=5 \mathrm{mag}$, estimated from the Weingartner \& Draine (2001) Milky Way model of $R_{V}=3.1$. The black line indicates our criterion (2).

\subsection{Colour-magnitude diagram}

Secondly, we can separate post-AGB stars, PNe, and galaxies from TTSs in the (UCAC $-J$ ) vs. (UCAC) colour-magnitude diagram. Owing to the low luminosities of PNe (white dwarfs) and galaxies at the visible wavelengths, we can remove about 90 and $97 \%$ of PNe and galaxies, respectively, by the applying the 2nd criterion

$$
\mathrm{UCAC} \leq 1.5 \times(\mathrm{UCAC}-J)+10 .
$$

From this criterion, we can also remove about $26 \%$ of post-AGB stars. Furthermore, 4 and $12 \%$ of carbon and OH/IR stars, respectively, can also be distinguished. Considering the detection limits of the IRC-PSC and the UCAC, this method seems effective only for extracting nearby $(\lesssim 100 \mathrm{pc})$ young sources. We note that we never identify TTSs with their edge-on disks, because they are not optically visible.

Figure 9 shows the same colour-colour diagrams as in Fig. 6, but for the remaining sources that could not be removed by the 

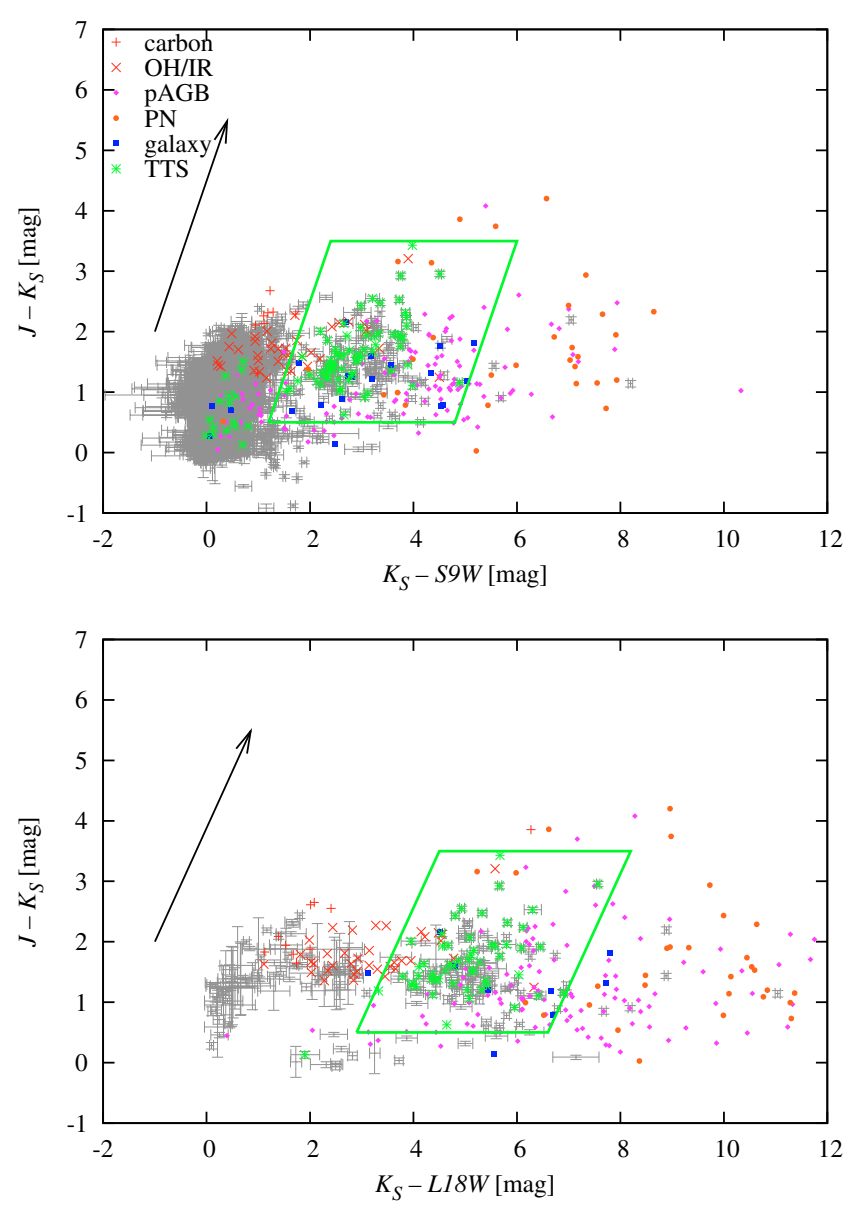

Fig. 9. Colour-colour diagrams, the same as in Fig. 6, but for the selected sources with the criteria (1) and (2) in the text. The green parallelograms indicates our criteria (3) and (4).

criteria (1) and (2); most of the remaining sources with the excess emission are the TTSs. Finally, we propose the following two criteria to select most of the TTSs affected by less contamination than other types of sources:

$$
\begin{gathered}
-11.5 \leq\left(J-K_{\mathrm{S}}\right)-2.5 \times\left(K_{\mathrm{S}}-S 9 W\right) \leq-2.5 \\
0.5 \leq J-K_{\mathrm{S}} \leq 3.5, \\
-12 \leq\left(J-K_{\mathrm{S}}\right)-1.9 \times\left(K_{\mathrm{S}}-S 9 W\right) \leq-5 \\
0.5 \leq J-K_{\mathrm{S}} \leq 3.5 .
\end{gathered}
$$

We note that we are unable to identify TTSs that have weak MIR excess emission, because they are contaminated by field stars. Consequently, most AGB stars, PNe, and galaxies are removed with our criteria. However, $34 \%$ of post-AGB stars remain, and we need to perform follow-up observations to differentiate young stars from post-AGB stars. Of the 133 AKARI-detected TTSs, 68 sources pass these criteria.

\section{Evaluation of the criteria}

\subsection{New TTS candidates from our criteria}

On the basis of our criteria, we selected 176 sources out of 14725 AKARI sources, which are distributed in the area between $2^{\mathrm{h}} 40^{\mathrm{m}}$ and $5^{\mathrm{h}} 40^{\mathrm{m}}$ in right ascension and between $0^{\circ}$ and $40^{\circ}$ in declination. We also searched for these sources in SIMBAD with a $10^{\prime \prime}$ searching radius. In the 176 selected sources, 21 sources could not be found in the SIMBAD database: there were 115 YSOs, 4 Be stars, 10 AGB stars, 1 galaxy, and 18 other types of objects (mostly variable stars), the remaining sources consisting of 7 unknown objects. Since $\sim 75 \%$ of the SIMBAD-identified sources were YSOs, we assume the TTS-identification probability based on our criteria to be $75 \%$, though that is of course affected by the completeness of the SIMBAD database.

We identified 21 new TTS candidates in addition to the 7 unknown sources. The infrared properties of the 28 TTS candidates are listed in Table 2. Considering the TTS-identification probability of $75 \%$, we would indeed expect to find 21 new TTSs. We need to perform follow-up observations to determine whether these sources are really TTSs or not.

\subsection{Comparison with other methods}

Many astronomers have discussed the criteria to extract YSO candidates from their surveyed data. Evans et al. (2009) and Rebull et al. (2010) surveyed YSOs in nearby clouds using Spitzer. Evans et al. (2009) surveyed 5 clouds (15.5 $\mathrm{deg}^{2}$ region in total) and listed $\sim 1000$ YSO candidates based on the colour-magnitude and colour-colour diagrams by 2MASS and Spitzer. They showed that there might be 51 galaxies among their YSO candidates. However, since their surveyed areas are highly embedded $\left(A_{V}>2\right)$ and located at high Galactic latitudes, they paid less attention to AGB stars. Rebull et al. (2010) surveyed a $\sim 44 \mathrm{deg}^{2}$ region of the Taurus molecular cloud, and listed 148 new candidate Taurus members based on colours and magnitudes drawn from the 2MASS and Spitzer datasets and the images of SDSS and CFHT. Of these 148 new candidates, they performed follow-up spectroscopic observations of about half of the sources, and confirmed 47 new Taurus candidates, 7 extragalactic objects, and 1 Be star; additional follow-up observations should also be performed for the remaining 93 sources.

These two studies have higher (973/1024, Evans et al. 2009; 47/55, Rebull et al. 2010) YSO-identification probabilities than our probability of $\sim 75 \%$. This is mainly because we have data for only 2 bands; they usually have more than 4 bands. Furthermore, because the detection limits of the AKARI AllSky Survey shallower than those of the pointed observations of Spitzer, it is difficult to detect MIR faint objects. However, since AKARI has observed almost the whole sky, we have beed able to identify TTS candidates towards all nearby star-forming regions.

Acknowledgements. This work is based on observations with AKARI, a JAXA project with the participants of ESA. We gratefully acknowledge all the members of the AKARI project for their support on this project. This publication makes use of data products from the Two Micron All Sky Survey, which is a joint project of the University of Massachusetts and the Infrared Processing and Analysis Center/California Institute of Technology, funded by the National Aeronautics and Space Administration and the National Science Foundation. This research has made use of the SIMBAD database and the VizieR catalogue access tool, CDS, Strasbourg, France. This research has made use of the NASA/IPAC Extragalactic Database (NED) which is operated by the Jet Propulsion Laboratory, California Institute of Technology, under contract with the National Aeronautics and Space Administration.

\section{References}

Acker, A., Ochsenbein, F., Stenholm, B., et al. 1994, VizieR Online Data Catalog, 5084, 0

Adams, F. C., Lada, C. J., \& Shu, F. H. 1988, ApJ, 326, 865

Beckwith, S. V. W., Sargent, A. I., Chini, R. S., \& Guesten, R. 1990, AJ, 99, 924 


\section{S. Takita et al.: Taurus TTSs with AKARI}

Beichman, C. A., Neugebauer, G., Habing, H. J., Clegg, P. E., \& Chester, T. J. 1988, Infrared Astronomical Satellite (IRAS) Catalogs and Atlases, NASA RP-1190

Carpenter, J. M., Bouwman, J., Mamajek, E. E., et al. 2009, ApJS, 181, 197

Cieza, L., Padgett, D. L., Stapelfeldt, K. R., et al. 2007, ApJ, 667, 308

Cohen, M., \& Kuhi, L. V. 1979, ApJS, 41, 743

Evans, N. J., Dunham, M. M., Jørgensen, J. K., et al. 2009, ApJS, 181, 321

Furlan, E., Hartmann, L., Calvet, N., et al. 2006, ApJS, 165, 568

Güdel, M., Briggs, K. R., Arzner, K., et al. 2007, A\&A, 468, 353

Harris, S., Clegg, P., \& Hughes, J. 1988, MNRAS, 235, 441

Harvey, P., Merín, B., Huard, T. L., et al. 2007, ApJ, 663, 1149

Hayashi, C., Nakazawa, K., \& Nakagawa, Y. 1985, Protostars and Planets II, 1100

Herbig, G. H., \& Bell, K. R. 1988, Third Catalog of Emission-Line Stars of the Orion Population, Lick Observatory Bulletin, 1111

Ishihara, D., Onaka, T., Kaneda, H., et al. 2007, PASJ, 59, S443

Ishihara, D., Onaka, T., Kataza, H., et al. 2010, A\&A, 514, A1

Ita, Y., Matsuura, M., Ishihara, D., et al. 2010, A\&A, 514, A2

Kenyon, S. J., Gómez, M., \& Whitney, B. A. 2008, Handbook of Star Forming Regions, Vol. I, 405

Kitamura, Y., Momose, M., Yokogawa, S., et al. 2002, ApJ, 581, 357

Le Bertre, T., Tanaka, M., Yamamura, I., \& Murakami, H. 2003, A\&A, 403, 943

Li, J. Z., \& Hu, J. Y. 1998, A\&AS, 132, 173

Luhman, K. L., Whitney, B. A., Meade, M. R., et al. 2006, ApJ, 647, 1180

Luhman, K. L., Mamajek, E. E., Allen, P. R., \& Cruz, K. L. 2009, ApJ, 703, 399
Luhman, K. L., Allen, P. R., Espaillat, C., Hartmann, L., \& Calvat, N. 2010, ApJS, 186, 111

Magazzù, A., Martín, E. L., Sterzik, M. F., et al. 1997, A\&AS, 124, 449

Murakami, H., Baba, H., Barthel, P., et al. 2007, PASJ, 59, S369

Neuhäuser, R., Sterzik, M. F., Schmitt, J. H. M. M., Wichmann, R., \& Krautter, J. 1995a, A\&A, 295, L5

Neuhäuser, R., Sterzik, M. F., Schmitt, J. H. M. M., Wichmann, R., \& Krautter, J. 1995b, A\&A, 297, 391

Neuhäuser, R., Sterzik, M. F., Schmitt, J. H. M. M., Wichmann, R., \& Krautter, J. 1995c, A\&A, 299, L13

Onaka, T., Yamamura, I., de Jong, T., et al. 1998, Ap\&SS, 255, 331

Onaka, T., Matsuhara, H., Wada, T., et al. 2007, PASJ, 59, S401

Padgett, D. L., Cieza, L., Stapelfeldt, K. R., et al. 2006, ApJ, 645, 1283

Rebull, L. M., Padgett, D. L., McCabe, C.-E., et al. 2010, ApJS, 186, 259

Silverstone, M. D., Meyer, M. R., Mamajek, E. E., et al. 2006, ApJ, 639, 1138

Skrutskie, M. F., Cutri, R. M., Stiening, R., et al. 2006, ApJ, 131, 1163

Strom, K. M., Strom, S. E., Edwards, S., Cabrit, S., \& Skrutskie, M. F. 1989, AJ, 97, 1451

Szczerba, R., Siódmiak, N., Stasińska, G., et al. 2007, A\&A, 469, 799

Tanabé, T., Sakon, I., Cohen, M., et al. 2008, PASJ, 60, S375

Weingartner, J. C., \& Draine, B. T. 2001, ApJ, 548, 296

Wichmann, R., Krautter, J., Schmitt, J. H. M. M., et al. 1996, A\&A, 312, 439

Wyatt, M. C., Dent, W. R. F., \& Greaves, J. S. 2003, MNRAS, 342, 876

Zacharias, N., Urban, S. E., Zacharias, M. I., et al. 2004, AJ, 127, 3043

Zacharias, N., Finch, C., Girard, T., et al. 2010, AJ, 139, 2184 